\title{
Spin-Orbit-Entangled Nature of Magnetic Moments and Kitaev Magnetism in Layered Halides $\alpha-\operatorname{RuX}_{3}(\mathrm{X}=\mathrm{Cl}, \mathrm{Br}, \mathrm{I})$
}

\author{
Received 19 November, 2021; accepted 24 November, 2021
}

\begin{abstract}
Heung-Sik Kim* (i)
Department of Physics and Institute for Accelerator Science, Kangwon National University, Chuncheon 24341, Republic of Korea
\end{abstract}

\section{*Corresponding author E-mail: heungsikim@kangwon.ac.kr}

\begin{abstract}
Recently, $\alpha-\mathrm{RuCl}_{3}$ has been extensively studied because of potential bond-dependent Kitaev magnetic exchange interactions and the resulting quantum spin liquid phase that can be realized therein. The covalency between $\mathrm{Ru} 4 d$ - and $\mathrm{Cl} p$-orbitals is crucial for inducing large Kitaev interactions in this compound, therefore replacing $\mathrm{Cl}$ with heavier halogen elements such as $\mathrm{Br}$ or I appears to be a promising method for further promoting the Kitaev interaction. There have been several reports on synthesis of $\alpha-\mathrm{RuBr}_{3}$ and $\alpha$ - $\mathrm{RuI}_{3}$, which are expected to host the same spin-orbit-entangled orbitals and Kitaev exchange interactions with $\alpha-\mathrm{RuCl}_{3}$. This study investigated electronic structures of $\alpha$ - $\mathrm{RuCl}_{3}$, $\alpha-\mathrm{RuBr}_{3}$, and $\alpha-\mathrm{RuI}_{3}$ via comparisons, focusing on the cooperation of the spin-orbit coupling and on-site Coulomb repulsions to realize the spin-orbit-entangled pseudospin-1/2 at Ru sites. In addition, magnetic exchange interactions of all three compounds were estimated, thereby demonstrating that $\alpha-\mathrm{RuBr}_{3}$ can be promising candidates for realizing Kitaev spin liquid phases in solid-state systems.
\end{abstract}

Keywords: Kitaev magnetism, Layered transition metal halides, Spin-orbit coupling, Strongly correlated electron systems, Frustrated magnetism, Density functional theory

\section{Introduction}

Kitaev's finding of an exactly solvable magnetic model on a twodimensional honeycomb lattice [1], which promises a fault-tolerant quantum computation via realization of Majorana fermions with nonAbelian statistics, ushered in a plethora of theoretical and experimental studies that aimed at realizing the Kitaev physics within condensed matter systems. A general guiding principle was reported by Jackeli and Khaliullin [2], followed by a number of theoretical and experimental studies that attempted to determine and evaluate the viability of the Jackeli-Khaliullin mechanism in realistic systems [3,4]. Currently, $\alpha-\mathrm{RuCl}_{3}$ is considered the most promising candidate [5-15], wherein half-quantized thermal Hall conductivity, considered as the essential evidence for the presence of the Kitaev spin liquid (KSL) state $[1,13,15]$, is reported.

However, attaining the KSL state in $\alpha-\mathrm{RuCl}_{3}$ is hindered by the presence of magnetic exchange interactions, in addition to Kitaev interactions, which induce static magnetism and disrupt the KSL phase. Specifically, the presence of the first- and third-nearest-neighbor Heisenberg interaction is detrimental for the Kitaev magnetism [16], and completely removing them is challenging owing to the multi-orbital nature of candidate systems and the resulting various hopping processes [17]. Therefore, selective enhancement of relevant electron hopping channels that contribute to the Kitaev's exchange interaction are essential for the realization of the KSL phase in realistic situations.

Recently, certain theoretical and experimental reports on $\alpha-\mathrm{RuBr}_{3}$ [18-20] and $\alpha-\mathrm{RuI}_{3}[19,21,22]$ have been presented, focusing on the possibility of promoting and realizing the KSL phase. As discussed in further detail later, the heavier ligand ion results in stronger hybridization between $\mathrm{Ru} 4 d$ - and ligand $(\mathrm{Cl}, \mathrm{Br}, \mathrm{I}) p$-orbitals, which may lead to stronger Kitaev interactions. However, it may also induce unwanted enhancement of further-neighbor and even inter-layer Heisenberg interactions, which are not favorable to realizing the KSL phases. Moreover, enhanced bandwidth due to larger $d$ - $p$ hybridization can also disturb the formation of the local spin-orbital-entangled $j_{\text {eff }}=1 / 2$ moments, a critical element of the Kitaev's exchange interactions. Therefore, evaluation of such quantities via first-principles electronic structure calculations is crucial during the early stage of studies regarding $\alpha-\mathrm{RuBr}_{3}$ and $\alpha-\mathrm{RuI}_{3}$.

Thus, this study focused on the electronic structure of $\alpha-\mathrm{RuBr}_{3}$ and $\alpha-\mathrm{RuI}_{3}$, comparisons with $\alpha-\mathrm{RuCl}_{3}$, and the formation of the spinorbital-entangled $j_{\text {eff }}=1 / 2$ moments in the compounds. Furthermore, the magnitudes of magnetic exchange interactions were estimated via employing first-principles Wannierization of $\mathrm{Ru} t_{2 g}$ bands, showing $\alpha-\mathrm{RuBr}_{3}$ to be a promising platform to further investigate the Kitaev magnetism in condensed matter systems.

\section{Computational details}

For the optimizations of cell parameters and internal coordinates of all three compounds, the Vienna ab-initio Simulation Package (VASP), which uses the projector-augmented wave (PAW) basis set $[23,24]$ was employed. $500 \mathrm{eV}$ of plane-wave energy curoff and $7 \times 7 \times 3 \Gamma$-centered $k$-grid sampling were adopted. Further, effects of electron correlations in structural optimizations were considered via the Strongly Constrained and Appropriately Normed (SCAN) semi-local functional [25], which is parameter-free, combined with a revised Perdew-Burke-Ernzerhof 
(a)

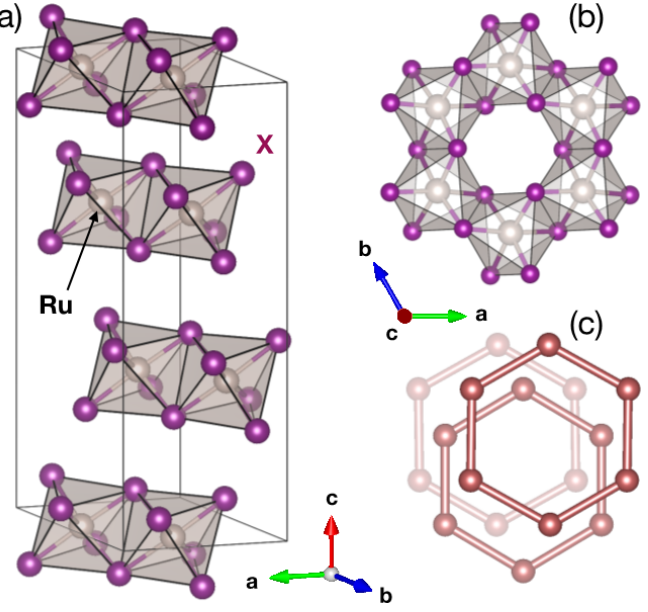

Figure 1. (a) Crystal structure of $\alpha-\mathrm{RuX}_{3}(\mathrm{X}=\mathrm{Cl}, \mathrm{Br}, \mathrm{I})$ with $\mathrm{P}_{1} 12$ space group. Solid lines depict the trigonal unit cell. (b) A top-down view of the single RuX $\mathrm{X}_{3}$ layer, where the stacking of neighboring Ru honeycomb layers are shown in (c).

(PBEsol) [26] exchange-correlation functional. Following the structural optimizations, effects of atomic spin-orbit coupling (SOC) and on-site Coulomb repulsions were studied employing a linear-combination-of-pseudo-atomic-orbital basis code OPENMX [27, 28], where projections onto the spin-orbit-entangled $j_{\text {eff }}=1 / 2$ and $3 / 2$ states and the Wannierization of $\mathrm{Ru} t_{2 g}$ bands were performed [29-31]. Double zeta plus polarization (DZP) bases and 300 Ry of energy cut-off for real space integrations were employed in the OPENMX calculations, and the effects of on-site Coulomb repulsions were incorporated via a simplified variant of the rotationally-invariant $\mathrm{DFT}+U$ formalism [32] combined with the local density approximation $[33,34]$. The value of $U_{\text {eff }} \equiv U-J$ was chosen as $2 \mathrm{eV}$, which has been considered to be a reasonable value in previous studies $[6,10]$. Electron hopping integrals were computed using Wannier orbital method in the nonmagnetic phase without including SOC and on-site Coulomb interactions, thereby eliminating the double-counting of such effects.

\section{Results and discussion}

\subsection{Crystal structures}

Figure 1 shows the model crystal structure we chose for $\alpha-\mathrm{RuX}_{3}$ (X $=\mathrm{Cl}, \mathrm{Br}, \mathrm{I}$ ) in this study (Space group: $\mathrm{P} 3_{1} 12$ ). There have been reports of different space group symmetries and stacking patterns for these compounds [20,22], but our choice of the $\mathrm{P} 3_{1} 12$ stacking is not a serious concern because interlayer interactions are fairly small in all three compounds [10]. Moreover, while the zigzag-type antiferromagnetic order (which requires larger in-plane periodicity) has been reported as the true ground state for $\alpha-\mathrm{RuCl}_{3}$, the effect of different antiferromagnetism within the $\mathrm{RuCl}_{3}$ layer on structural and electronics properties is insignificant. Therefore, a Néel-type antiferromagnetism was employed in our structural optimizations.

Table I presents PBEsol+SCAN-optimized lattice parameters of all three compounds in the presence of SOC. Our result predicted lattice parameters of $\alpha-\mathrm{RuCl}_{3}$ and $\alpha-\mathrm{RuI}_{3}$ to be approximately $1 \%$ larger than experimentally reported values $[22,35]$; however, the tendency toward cell expansion with the ligand ion becoming heavier was well-captured

Table I. Lattice parameters of $\alpha-\mathrm{RuCl}_{3}, \alpha-\mathrm{RuBr}_{3}$, and $\alpha-\mathrm{RuI}_{3}$ from PBEsol+SCAN optimizations.

\begin{tabular}{c|c|c}
\hline (in $\AA$ ) & $|\mathrm{a}|$ & $|\mathrm{c}|$ \\
\hline$\alpha-\mathrm{RuCl}_{3}$ & 6.043 & 17.601 \\
$\alpha-\mathrm{RuBr}_{3}$ & 6.404 & 18.634 \\
$\alpha-\mathrm{RuI}_{3}$ & 6.888 & 20.236 \\
\hline
\end{tabular}
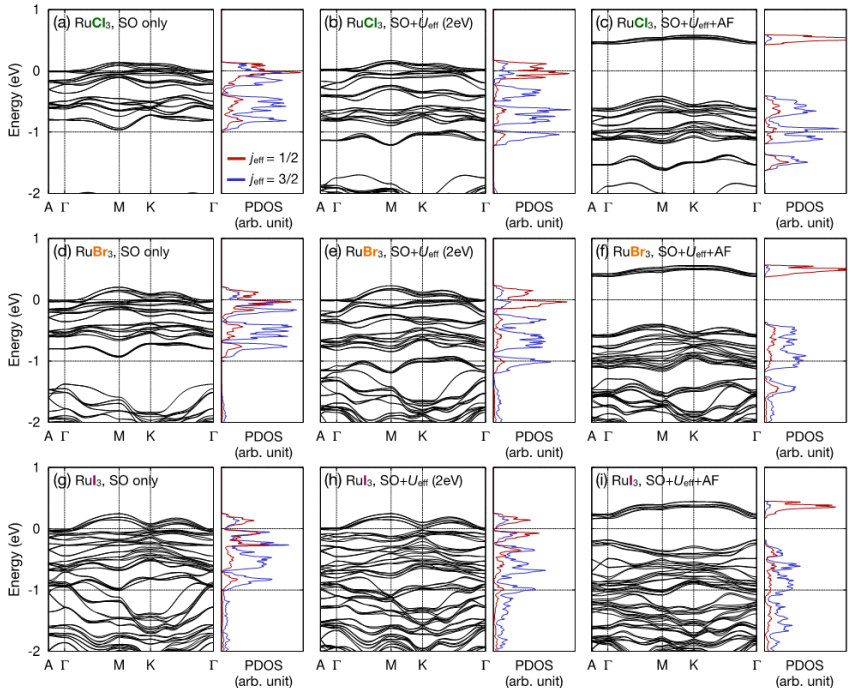

Figure 2. Band structures and projected densities of states (PDOS) onto the Ru $j_{\text {eff }}=1 / 2$ and $3 / 2$ states. Top $(a-c)$, middle $(d-f)$, and bottom (g-i) rows show results obtained for $\alpha-\mathrm{RuCl}_{3}, \alpha-\mathrm{RuBr}_{3}$, and $\alpha-\mathrm{Rul}_{3}$, respectively. Left (a, d, and g), middle ( $b, e$, and $h$ ), and right (c, f, and i) columns show results with SOC, SOC $+U_{\text {eff }}(2$ $\mathrm{eV}$ ), and $\mathrm{SOC}+U_{\text {eff }}+$ antiferromagnetism (Neel type), respectively. Note that nonmagnetic constraint was enforced when obtaining results in the left and middle columns.

within our results.

\subsection{Electronic structure and spin-orbital-entangled states}

Figure 2 summarizes our calculation results for all three compounds, showing the band structures and projected densities of states (PDOS) onto the spin-orbit-entangled $j_{\text {eff }}=1 / 2$ and $3 / 2$ states. The Ru $j_{\text {eff }}$ states, eigenstates of the SOC Hamiltonian within the $\mathrm{Ru} t_{2 g}$ orbital, are defined as

$$
\begin{aligned}
& \left|j_{\text {eff }}=\frac{1}{2} ; \pm \frac{1}{2}\right\rangle=\frac{1}{\sqrt{3}}\left(\left|d_{x y}, \uparrow \downarrow\right\rangle \pm\left|d_{y z}, \downarrow \uparrow\right\rangle+i\left|d_{x z}, \downarrow \uparrow\right\rangle\right), \\
& \left|j_{\text {eff }}=\frac{3}{2} ; \pm \frac{1}{2}\right\rangle=\sqrt{\frac{2}{3}}\left(\left|d_{x y}, \uparrow \downarrow\right\rangle \mp \frac{\left|d_{y z}, \downarrow \uparrow\right\rangle \pm i\left|d_{x z}, \downarrow \uparrow\right\rangle}{2}\right), \\
& \left|j_{\text {eff }}=\frac{3}{2} ; \pm \frac{3}{2}\right\rangle=\mp \frac{1}{\sqrt{2}}\left(\left|d_{y z}, \uparrow \downarrow\right\rangle \pm i\left|d_{x z}, \uparrow \downarrow\right\rangle\right) .
\end{aligned}
$$

On comparing the left and right columns in Fig. 2, it is evident that the inclusion of the on-site Coulomb interaction enhanced the splitting between the $j_{\text {eff }}=1 / 2$ and $3 / 2$ states. The SOC being effectively enhanced by $U_{\text {eff }}$, has been discussed in previous studies regarding $\alpha$ $\mathrm{RuCl}_{3}[6,22]$. Further, the inclusion of magnetism and the opening of the band gap further enhances the splitting (comparing the middle and right columns in Fig. 2), consequently driving $\alpha-\mathrm{RuCl}_{3}$ and $\alpha-\mathrm{RuI}_{3}$ good Kitaev magnet candidates. However, in contrast to the results obtained in our study [Fig. 2(a)] $\alpha-\mathrm{RuI}_{3}$ has been reported to be metallic [21]. The discrepancy may originate from technical details (e.g., the choice of local atomic projectors employed in the $\mathrm{DFT}+U_{\text {eff }}$ methodology). We comment that, the results of this study indicate that the band gap size of $\alpha-\mathrm{RuI}_{3}$ is much smaller than that of $\alpha-\mathrm{RuCl}_{3}$ and $\alpha-\mathrm{RuBr}_{3}$ [comparing Figs. 2(c), 2(f), and 2(i)], which is consistent with the fact that larger $d-p$ hybridization in $\alpha-\mathrm{RuI}_{3}$ induces larger bandwidth and weaker electron correlation effects. In addition, the enhancement of the $j_{\text {eff }}=1 / 2-3 / 2$ splitting introduced by $U_{\text {eff }}$ is not significant [Figs. 2(g) and 2(h)] compared to other compounds, possibly because of the larger $d$ - $p$ hybridization in $\alpha-\mathrm{RuI}_{3}$. 


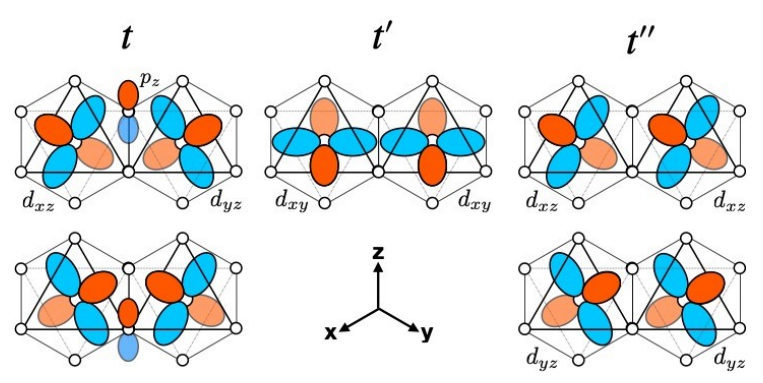

Figure 3. Illustration of major hopping channels between nearest-neighboring Ru $t_{2 g}$ orbitals.

\subsection{Electron hopping channels and magnetic excha- nge interactions}

Having established the spin-orbit-coupled nature of our systems, we next switch to magnetic exchange interactions. Figure 3 represents major nearest-neighbor hopping channels between $\mathrm{Ru} \mathrm{t} \mathrm{t}_{2 \mathrm{~g}}$ orbitals. Among these the first t-term, which is mediated via an intermediate anion p-orbital, is critical to inducing the Kitaev interaction, whereas the remaining terms result in conventional Heisenberg or symmetric anisotropy interactions. Specifically, the general magnetic Hamiltonian in $\alpha-\mathrm{RuX}_{3}$ is approximately expressed as [17],

$$
\begin{aligned}
H & \cong \sum_{\langle i j\rangle \in \alpha \beta(\gamma)}\left(J \vec{S}_{i} \cdot \vec{S}_{j}+K S_{i}^{\gamma} S_{j}^{\gamma}+\Gamma\left(S_{i}^{\alpha} S_{j}^{\beta}+S_{i}^{\beta} S_{j}^{\alpha}\right)\right) \\
& +\sum_{\langle\langle\langle i j\rangle\rangle\rangle \in \alpha \beta(\gamma)} J_{3} \vec{S}_{i} \cdot \vec{S}_{j},
\end{aligned}
$$

where $\alpha, \beta, \gamma$ denote bond directions and the relevant spin components $(x, y, z)$ involved in the Kitaev interactions, respectively, and $\langle i j\rangle$ and $\langle\langle\langle i j\rangle\rangle\rangle$ indicate the nearest-neighbor and third-nearest-neighbor $i-j$ sites, respectively. The $J, K$, and $\Gamma$ are explicitly obtained via perturbation theory as follows [17],

$$
\begin{aligned}
& J=\frac{4}{27}\left[\frac{6 t^{\prime \prime}\left(t^{\prime \prime}+2 t^{\prime}\right)}{U-3 J_{H}}+\frac{2\left(t^{\prime \prime}-t^{\prime}\right)^{2}}{U-J_{H}}+\frac{\left(2 t^{\prime \prime}+t^{\prime}\right)^{2}}{U+2 J_{H}}\right], \\
& K=\frac{8 J_{H}}{9}\left[\frac{\left(t^{\prime \prime}-t^{\prime}\right)^{2}-3 t^{2}}{\left(U-3 J_{H}\right)\left(U-J_{H}\right)}\right], \\
& \Gamma=\frac{16 J_{H}}{9}\left[\frac{t\left(t^{\prime \prime}-t^{\prime}\right)}{\left(U-3 J_{H}\right)\left(U-J_{H}\right)}\right],
\end{aligned}
$$

where $U$ and $J_{H}$ are the strength of the on-site Coulomb repulsion (not necessarily the same as $U_{\text {eff }}$ ) and Hund's coupling within the Ru $t_{2 g}$ orbital, respectively.

The hopping terms $t, t^{\prime}$, and $t^{\prime \prime}$ can be obtained using the Wannier function method $[29,30]$, which are tabulated in Table II. The magnitude of the $d-p$ - $d$ hopping term $t$ decreased slightly when changing from $\alpha-\mathrm{RuCl}_{3}$ to $\alpha-\mathrm{RuI}_{3}$. In addition, the magnitudes of $t^{\prime}$ and $t^{\prime \prime}$, which introduce Heisenberg $J$, decreased when $\mathrm{Cl}$ was replaced with $\mathrm{Br}$ or I. The origin of this behavior is unclear at this point and deserves further study.

Because we obtained the hopping parameters from ab-initio calculations, the magnitudes of $J, K$, and $\Gamma$ could be estimated as a function

Table II. Activation energies and errors for various CdTe film thicknesses.

\begin{tabular}{c|c|c|c|c|c}
\hline$($ in $\mathrm{eV})$ & $t$ & $t^{\prime}$ & $t^{\prime \prime}$ & $t_{3 \mathrm{rd}}$ & $t_{\text {inter }}^{\max }$ \\
\hline$\alpha-\mathrm{RuCl}_{3}$ & +0.184 & -0.054 & +0.035 & -0.041 & -0.028 \\
$\alpha-\mathrm{RuBr}_{3}$ & +0.169 & -0.030 & +0.024 & -0.040 & -0.040 \\
$\alpha-\mathrm{RuI}_{3}$ & +0.170 & +0.007 & +0.009 & -0.050 & -0.049 \\
\hline
\end{tabular}

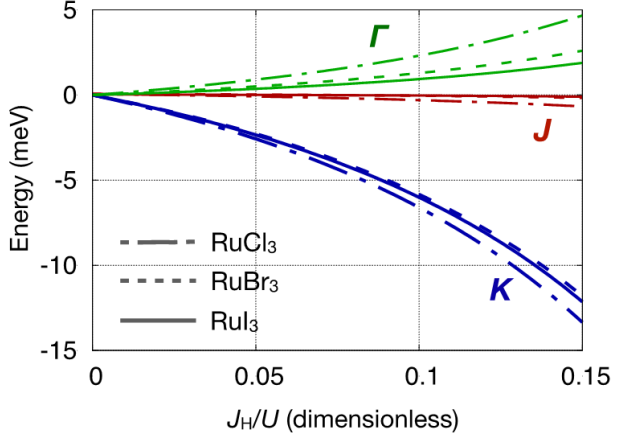

Figure 4. Nearest-neighbor magnetic exchange interactions for $\alpha-\mathrm{RuCl}_{3}, \alpha$ $\mathrm{RuBr}_{3}$, and $\alpha-\mathrm{Rul}_{3}$ as a function of $J_{H} / U$

of $U$ and $J_{H}$. We set $U=U_{\text {eff }}=2 \mathrm{eV}$, and computed exchange interactions as a function of $J_{H} / U$. Figure 4 shows the results, and owing to the diminishing $t^{\prime}$ and $t^{\prime \prime}$ in $\alpha-\mathrm{RuBr}_{3}$ and $\alpha-\mathrm{RuI}_{3}$, the size of $J$ and $\Gamma$ reduced when changing from $\alpha-\mathrm{RuCl}_{3}$ to $\alpha-\mathrm{RuI}_{3}$. As $\alpha-\mathrm{RuI}_{3}$ has been reported to be metal, $\alpha-\mathrm{RuBr}_{3}$ appears to be the best candidate to host Kitaev magnetism in this series of compounds.

We conclude this section by mentioning third-nearest-neighbor and inter-layer hopping elements. Table II indicates that both terms increased as the ligand anion became heavier, as expected in the beginning. However, the enhancement is not that significant compared to the changes in $t^{\prime}$ and $t^{\prime \prime}$. Although many different channels can constructively contribute to further neighbor and interlayer hopping channels, as discussed in Winter et al. [16], and Catuneanu et al. [36], it can be speculated that the enhancement in further neighbor and interlayer hopping channels may not cause substantial changes in $\alpha$ $\mathrm{RuBr}_{3}$ and $\alpha-\mathrm{RuI}_{3}$, thereby rendering $\alpha-\mathrm{RuBr}_{3}$ a promising candidate for realizing Kitaev magnetism in this family.

\section{Conclusions}

In this study preliminary first-principles density functional theory calculations were performed for $\alpha-\mathrm{RuCl}_{3}, \alpha-\mathrm{RuBr}_{3}$, and $\alpha-\mathrm{RuI}_{3}$, to assess their viability for realizing Kitaev magnetism. The results indicated that $\alpha-\mathrm{RuCl}_{3}$ and $\alpha-\mathrm{RuBr}_{3}$ may host the spin-orbit-entangled $j_{\text {eff }}=1 / 2$ moment, and that $\alpha-\mathrm{RuBr}_{3}$ shows even stronger Kitaev magnetism compared to $\alpha-\mathrm{RuCl}_{3}$.

Note that some possible effects of minor lattice distortions, for example the effect of trigonal crystal fields, were not discussed in this study [37]. Estimation of next- and third-nearest-neighbor Heisenberg interactions that induce long-range magnetic orders needs to be done as well for further studies on magnetic properties of these systems. Hence continuing theoretical and experimental studies are needed at this moment, but we believe that $\alpha-\mathrm{RuBr}_{3}$ can be another interesting system to study Kitaev physics in addition to $\alpha-\mathrm{RuCl}_{3}$. Lastly, since $\alpha-\mathrm{RuI}_{3}$ has been reported to be nonmagnetic and metallic, it has potentials to host topological band insulating phases with weakto-intermediate electron correlations as previously suggested [36].

\section{Acknowledgements}

We thank K.-Y. Choi for useful discussions, and acknowledge the support from the Korea Research Fellow (KRF) Program and the Basic Science Research Program through the National Research Foundation of Korea funded by the Ministry of Education (NRF-2019H1D3A1A01102984 and NRF- 2020R1C1C1005900). We also thank the support of computational resources including technical assistance from the $\mathrm{Na}$ tional Supercomputing Center of Korea (Grant No. KSC- 2020-CRE0156)

\section{Conflicts of Interest}

The authors declare no conflicts of interest. 


\section{ORCID}

Heung-Sik Kim

https://orcid.org/0000-0003-0691-7605

\section{References}

[1] A. Kitaev, Ann. Phys. 321, 2 (2006).

[2] G. Jackeli and G. Khaliullin, Phys. Rev. Lett. 102, 017205 (2009).

[3] S. M. Winter, A. A. Tsirlin, M. Daghofer, J. van den Brink, Y. Singh, P. Gegenwart, and R. Valenti, J. Phys. Condens. Matter 29, 493002 (2017).

[4] H. Takagi, T. Takayama, G. Jackeli, G. Khaliullin, and S. E. Nagler, Nat. Rev. Phys. 1, 264 (2019).

[5] K. W. Plumb, J. P. Clancy, L. J. Sandilands, V. Vijay Shankar, Y. F. Hu, K. S. Burch, H.-Y. Kee, and Y.-J. Kim, Phys. Rev. B 90, $041112(2014)$

[6] H.-S. Kim, V. Vijay Shankar, A. Catuneanu, and H.-Y. Kee, Phys. Rev. B 91, 241110 (2015).

[7] J. A. Sears, M. Songvilay, K. W. Plumb, J. P. Clancy, Y. Qiu, Y. Zhao, D. Parshall, and Y.-J. Kim, Phys. Rev. B 91, 144420 (2015).

[8] L. J. Sandilands, Y. Tian, K. W. Plumb, Y.-J. Kim, and K. S. Burch, Phys. Rev. Lett. 114, 147201 (2015)

[9] L. J. Sandilands, Y. Tian, A. A. Reijnders, H.-S. Kim, K. W. Plumb, Y.-J. Kim, H.-Y. Kee, and K. S. Burch, Phys. Rev. B 93, 075144 (2016).

[10] H.-S. Kim and H.-Y. Kee, Phys. Rev. B 93, 155143 (2016).

[11] A. Banerjee et al., Nat. Mater. 15, 733 (2016).

[12] S.-H. Do et al., Nat. Phys. 13, 1079 (2017).

[13] Y. Kasahara et al., Nature 559, 227 (2018).

[14] J. A. Sears, L. E. Chern, S. Kim, P. J. Bereciartua, S. Francoual, Y. B. Kim, and Y.-J. Kim, Nat. Phys. 16, 837 (2020).

[15] T. Yokoi et al., Science 373, 568 (2021).

[16] S. M. Winter, Y. Li, H. O. Jeschke, and R. Valenti, Phys. Rev. B 93, 214431 (2016).

[17] J. G. Rau, E. K.-H. Lee, and H.-Y. Kee, Phys. Rev. Lett. 112
077204 (2014).

[18] M. Salavati, N. Alajlan, and T. Rabczuk, Phys. E: Low-Dimens. Syst. Nanostructures 113, 79 (2019).

[19] F. Ersan, E. Vatansever, S. Sarikurt, Y. Yüksel, Y. Kadioglu, H. D. Ozaydin, O. Ü. Aktürk, Ü. Akinci, and E. Aktürk, J. Magn. Magn. Mater. 476, 111 (2019).

[20] Y. Imai et al., arXiv:2109.00129 (2021).

[21] D. Ni, X. Gui, K. M. Powderly, and R. J. Cava, arXiv:2108.12915 (2021).

[22] Y. Zhang, L.-F. Lin, A. Moreo, and E. Dagotto, arXiv:2111.04560 (2021).

[23] G. Kresse and J. Hafner, Phys. Rev. B 47, 558 (1993).

[24] G. Kresse and J. Furthmüller, Phys. Rev. B 54, 11169 (1996).

[25] J. Sun, A. Ruzsinszky, and J. P. Perdew, Phys. Rev. Lett. 115, 036402 (2015).

[26] J. P. Perdew, A. Ruzsinszky, G. I. Csonka, O. A. Vydrov, G. E. Scuseria, L. A. Constantin, X. Zhou, and K. Burke, Phys. Rev. Lett. 100, 136406 (2008).

[27] T. Ozaki, Phys. Rev. B 67, 155108 (2003).

[28] M. J. Han, T. Ozaki, and J. Yu, Phys. Rev. B 73, 045110 (2006).

[29] N. Marzari and D. Vanderbilt, Phys. Rev. B 56, 12847 (1997).

[30] I. Souza, N. Marzari, and D. Vanderbilt, Phys. Rev. B 65, 035109 (2001).

[31] H. Weng, T. Ozaki, and K. Terakura, Phys. Rev. B 79, 235118 (2009).

[32] S. L. Dudarev, G. A. Botton, S. Y. Savrasov, C. J. Humphreys, and A. P. Sutton, Phys. Rev. B 57, 1505 (1998).

[33] D. M. Ceperley and B. J. Alder, Phys. Rev. Lett. 45, 566 (1980).

[34] J. P. Perdew and A. Zunger, Phys. Rev. B 23, 5048 (1981).

[35] R. D. Johnson et al., Phys. Rev. B 92, 235119 (2015).

[36] A. Catuneanu, H.-S. Kim, O. Can, and H.-Y. Kee, Phys. Rev. B 94, 121118 (2016).

[37] J. G. Rau and H.-Y. Kee, arXiv:1408.4811 (2014). 